\title{
ON JACOBI'S ARITHMETICAL THEOREMS CONCERNING THE SIMULTANEOUS REPRESENTATION OF NUMBERS BY TWO DIFFERENT QUADRATIC FORMS*
}

\author{
BY \\ J. V. USPENSKY
}

\section{PART I}

The infinite series and products afforded by the theory of elliptic functions lead in a natural and easy way to the discovery of many peculiar arithmetical theorems which, at first sight, do not seem to be easily obtainable by purely arithmetical methods. But deeper insight into arithmetical properties of elliptic series and products shows that the use of them may be superseded by certain arithmetical relations of a very general nature. Liouville was the first to call attention to this fact, but he never attempted to give a complete and systematic account of his ideas, and for this reason they did not attract the attention they deserve. The author of this paper, by his personal investigations concerning this subject, has been led to the conclusion that all the results previously obtained by means of elliptic functions may be as well established by purely arithmetical methods of extremely elementary nature, and he published his investigations, so far as it was possible under the circumstances, in a series of papers. $\dagger$ It is his intention to show in this paper how the arithmetical theorems obtained by Jacobi in his memoir Ueber diejenigen unendlichen Reihen, deren Exponenten zugleich in zwei verschiedenen quadratischen Formen enthalten sind $\ddagger$ can be easily derived by very simple arithmetical considerations. The paper is divided into two parts, according to two different methods of treatment for questions of this kind. The method developed in the first part is based on Liouville's ideas, but,

* Presented to the Society, December 31, 1926; received by the editors January 12, 1927.

$\dagger \mathrm{J}$. Uspensky, On arithmetical theorems given by Stieltjes (in Russian), Bulletin of the Mathematical Society in Kharkov, vol. 13 (1912); On the representation of numbers by sums of squares (in Russian), ibid., vol. 13 (1912); On certain arithmetic theorems (in Russian), ibid., vol. 13 (1913); On the possibility of representation of primes by certain quadratic forms (in Russian), Bulletin of the Mathematical Society in Kazan, 1915; On the representation of numbers by the quadratic forms with 4 and 6 variables (in Russian), Bulletin of the Mathematical Society in Kharkov, vol. 16 (1916); Sur les relations entre les nombres des classes des formes quadratiques binaires et positives, Parts $1,2,3$, 4, 5, Bulletin of the Academy of Sciences of the U. S. S. R., 1925-26; Note sur le nombre de representations des nombres par une somme d'un nombre pair de carres, ibid., 1925.

$\$$ Jacobi's Gesammelte Werke, vol. 3, p. 220. 
though simple and elegant, it is not the best adapted to this subject. Such a method, meeting all the requirements of simplicity and efficacy, will be developed in the second part.

1. The fundamental identity and its consequences. Instead of using various properties and transformations of elliptic series and products, we shall take for our starting point a single very general formula which, in a certain sense, contains the very essence of the arithmetical properties of elliptic functions. Let $F(x, y, z)$ represent a function defined for integral values of the variables $x, y, z$ and satisfying the conditions

$$
\begin{aligned}
F(-x, y, z) & =-F(x, y, z), \quad F(x,-y,-z)=F(x, y, z), \\
F(0, y, z) & =0 .
\end{aligned}
$$

For any such function and for any positive integer $n$, the following fundamental relation holds:

$$
2 \sum F(\delta-2 i, d+i, 2 d+2 i-\delta)-\sum F(d+\delta, i, d-\delta)=T,
$$

where both sums extend over all the solutions of the indeterminate equation

$$
n=i^{2}+d \delta
$$

in integers $i, d, \delta$, the two last being supposed positive, and $T=0$ when $n$ is not a perfect square,

$$
T=\sum_{j=1}^{2 s-1}[2 F(2 s-j, s, 2 s-j)-F(2 s, s-j, 2 s-2 j)] \text { when } n=s^{2}, s>0 .
$$

In order to avoid lengthy explanations, it is useful to introduce the symbolic notation $\{A\}$ for a quantity related to an integer $n$, having value 0 except when $n$ is a perfect square: $n=s^{2}, s>0$, in which case the above symbol represents the number $A$. With this notation adopted, the fundamental equation (2) may be written as follows:

$$
2 \sum_{(a)}[F(\delta-2 i, d+i, 2 d+2 i-\delta)-F(d+\dot{\delta}, i, d-\delta)]
$$

(a)

$$
=\left\{\sum_{j=1}^{2 s-1}[2 F(2 s-j, s, 2 s-j)-F(2 s, s-j, 2 s-2 j)]\right\}, n=s^{2}, s>0,
$$

The proof of this important equation is very easy, and the reader can find it for instance in the first part of our memoir Sur les relations entre les nombres des classes des formes quadratiques binaires et positives. $\dagger$ * In this formula (a) $n=i^{2}+d \delta$ indicates the range of summation for $\sum_{(a)}$. A similar notation
is used throughout.

† Loc. cit. 
From the fundamental equation (2), by submitting the highly arbitrary function $F(x, y, z)$ to certain restrictions, many other important relations may be derived. In this paper, however, we confine ourselves only to such of these relations as are absolutely necessary for our purpose. Denoting by $f(x)$ an arbitrary odd function of a single variable $x$, we can first take, in the fundamental equation (2),

$$
F(x, y, z)=0 \text { for even } x, \quad F(x, y, z)=f(x) \text { for odd } x,
$$

which, after a simple discussion, leads to the following relation:

$$
\sum_{(a)} f(2 d+\delta)=\sum_{(b)} f(\delta-2 i)-\left\{\sum_{(c)} f(j)\right\}
$$

(a) $n=i^{3}+2 d \delta$, $\delta$ odd;

(b) $n=i^{2}+d \delta$. $\delta$ odd :

(c) $j=1,3,5, \cdots, 2 s-1, n=s^{2}, \quad s>0$.

Again, assuming in the same fundamental equation

$$
\begin{aligned}
& F(x, y, z)=0 \text { for even } x, \\
& F(x, y, z)=(-1)^{(x+z) / 2+y} f(y) \text { for odd } x,
\end{aligned}
$$

we arrive at the very useful formula

$$
\begin{gathered}
\sum_{(a)}(-1)^{i} f(d+i)=\left\{(-1)^{r-1} s f(s)\right\}, n=s^{2}, s>0, \\
n=i^{2}+d \delta, \delta \text { odd. }
\end{gathered}
$$

Suppose now $n$ divisible by 4 , so that $n=4 m$. By taking first for $F(x, y, z)$ the function defined as follows:

$$
\begin{aligned}
& F(x, y, z)=0, \text { whenever } x \text { is odd, } \\
& F(x, y, z)=0, \text { whenever } y \text { is even, } \\
& F(x, y, z)=\phi(x / 4, y), \text { otherwise, }
\end{aligned}
$$

where $\phi(x, y)$ denotes an arbitrary function odd with respect to $x$ and even with respect to $y$, we have after a simple discussion and with a slightly changed notation

$$
\sum_{(a)} \phi[(d+\delta) / 4, \lambda]=2 \sum_{(b)} \phi(d+i, \delta-2 i)+\left\{2 \sum_{(c)} \phi(s, j)\right\},
$$

(a) $\quad 4 m=\lambda^{2}+d \delta, \lambda$ odd;

(b) $m=i^{2}+d \delta, 8$ odd;

(c) $j=1,3,5, \cdots, 2 s-1, m=s^{2}, s>0$.

Taking again

$$
\begin{aligned}
& F(x, y, z)=0, \text { whenever } x \text { is odd, } \\
& F(x, y, z)=0, \text { whenever } y \text { is even, } \\
& F(x, y, z)=(-1)^{(y-z / 2) / 2} \phi(x / 4, y), \text { otherwise, }
\end{aligned}
$$


we get the equation

$$
\begin{aligned}
\sum_{(a)}(-1)^{(\lambda-1) / 2+(d-\delta-2) / 4} \phi & {[(d+\delta) / 4, \lambda]=2 \sum_{(b)}(-1)^{d} \phi(d+i, \delta-2 i) } \\
+ & \left\{2 \sum_{(c)} \phi(s, j)\right\},
\end{aligned}
$$

(a) $4 m=\lambda^{2}+d \delta, \lambda$ odd;

(b) $m=i^{2}+d \delta, \delta$ odd;

(c) $j=1,3,5, \cdots, 2 s-1, \quad m=s^{2}, s>0$,

the left member of which is obviously equal to 0 . Subtracting now (7) from (6) we have finally

$$
\sum_{(a)} \phi[(d+\delta) / 4, \lambda]=4 \sum_{(b)} \phi(d+i, \delta-2 i),
$$

(a) $4 m=\lambda^{2}+d \delta, \lambda$ odd;

(b) $m=i^{2}+d \delta, d$ and $\delta$ odd.

The last equation we need may be obtained as follows. First we suppose $n$ divisible by 3 , so that $n=3 m$, and then define $F(x, y, z)$ as follows:

$$
\begin{aligned}
& F(x, y, z)=0, \text { whenever } y+z \text { is not divisible by } 3, \\
& F(x, y, z)=0, \text { whenever } y \text { is divisible by } 3, \\
& F(x, y, z)=f(x / 3) \text { otherwise, }
\end{aligned}
$$

$f(x)$ being an arbitrary odd function of $x$. It is obvious that this definition is consistent with the fundamental conditions (1). The resulting equation with a slightly modified notation may be written as follows:

$$
\sum_{(a)} f\left[\left(\Delta+\Delta^{\prime}\right) / 3\right]=2 \sum_{(b)} f(\delta-2 i)+\{4 s f(2 s)\}, m=3 s^{2}, s>0,
$$

(a) $3 m=h^{2}+\Delta \Delta^{\prime}, h+\Delta-\Delta^{\prime} \equiv 0 \quad(\bmod 3)$;

(b) $m=3 i^{2}+d \delta, d \neq 0(\bmod 3)$,

and admits of a further simplification. As $h$ is supposed to be non-divisible by 3 in the equation $3 m=h^{2}+\Delta \Delta^{\prime}$, it is easy to see that, whenever $h+\Delta-\Delta^{\prime}$ is divisible by $3,-h+\Delta-\Delta^{\prime}$ is not divisible by 3 , and, whenever $h+\Delta-\Delta^{\prime}$ is not divisible by $3,-h+\Delta-\Delta^{\prime}$ is divisible by 3 , whence it follows that

$$
\sum_{(a)} f\left[\left(\Delta+\Delta^{\prime}\right) / 3\right]=\sum_{(b)} f\left[\left(\Delta+\Delta^{\prime}\right) / 3\right]
$$

(a) $h+\Delta-\Delta^{\prime} \equiv 0, h \neq 0 \quad(\bmod 3) ; \quad$ (b) $h+\Delta-\Delta^{\prime} \neq 0, h \neq 0(\bmod 3)$,

or

$$
2 \sum_{(a)} f\left[\left(\Delta+\Delta^{\prime}\right) / 3\right]=\sum_{(b)} f\left[\left(\Delta+\Delta^{\prime}\right) / 3\right]
$$
(a) $h+\Delta-\Delta^{\prime}=0, h \neq 0 \quad(\bmod 3)$;
(b) $m=h^{2}+\Delta \Delta^{\prime}, k \neq 0(\bmod 3)$. 
The left hand member of (9) being thus simplified, we finally get the important relation

$$
\sum_{(a)} f\left[\left(\Delta+\Delta^{\prime}\right) / 3\right]=4 \sum_{(b)} f(\delta-2 i)+\{8 s f(2 s)\}, m=3 s^{2}, s>0,
$$
(a) $3 m=h^{2}+\Delta \Delta^{\prime}, h \not \equiv 0 \quad(\bmod 3)$;
(b) $3 m=3 i^{2}+d \delta d \neq 0(\bmod 3)$.

2. Number of representations by certain quadratic forms. In the following discussion we need to use the well known results concerning the number of representations by quadratic forms $x^{2}+y^{2}, x^{2}+2 y^{2}$ and $x^{2}+3 y^{2}$. The discussion would be considerably abbreviated should we simply recall these results, but, from a methodical point of view, it is of interest to derive all the auxiliary propositions from one and the same source.

Beginning with the quadratic form $x^{2}+y^{2}$, let us consider the equation $n=x^{2}+y^{2}$, and denote by $N(n)$ the number of its solutions. The following two equations are obvious:

$$
\begin{aligned}
N(4 n) & =N(n), \\
N(n) & =0, \text { when } n \equiv 3
\end{aligned}
$$

$(\bmod 4)$.

Furthermore, denoting by $m$ any odd number, let us consider the equation

$$
2 m=x^{2}+y^{2}+z^{2} \text {. }
$$

We do not assume that this equation is necessarily solvable, but, if it is, among the numbers $x, y, z$ there are two odd and one even. The number of solutions with an odd $x$ is, therefore, twice as great as the number of solutions with an even $x$, which leads to the recurrent relation

$$
\sum_{(a)} N\left(2 m-x^{2}\right)=2 \sum_{(b)} N\left(2 m-4 x^{2}\right),
$$

(a) $x= \pm 1, \pm 3, \pm 5, \cdots$;

(b) $x=0, \pm 1, \pm 2, \cdots$,

both series being continued so far as the arguments remain positive. The same relation may be also written in the form

$$
\begin{gathered}
\sum_{(a)}(-1)^{x} N\left(2 m-x^{2}\right)+\sum_{(a)} N\left(2 m-4 x^{2}\right)=0, \\
x=0, \pm 1, \pm 2, \cdots .
\end{gathered}
$$

If there are no representations of $2 m$ by the sum of three squares (which is really impossible), both the sums in (11) are equal to 0 , and (11) continues to hold.

Considering the equation $m=x^{2}+y^{2}+z^{2}$, and supposing first $m=1$ 
( $\bmod 4)$, we easily see that the number of solutions with an even $x$ is twice as great as the number of solutions with an odd $x$, so that

$$
\sum_{(a)} N\left(m-4 x^{2}\right)=2 \sum_{(b)} N\left(m-x^{2}\right),
$$
(a) $x=0, \pm 1, \pm 2, \cdots$;
(b) $x= \pm 1, \pm 3, \pm 5, \cdots$,

or, what is the same thing,

$$
\sum_{(a)} N\left(m-x^{2}\right)=\sum_{(b)}(-1)^{x} N\left(m-x^{2}\right),
$$

(a) $x= \pm 1, \pm 3, \pm 5, \cdots$;

(b) $x=0, \pm 1, \pm 2, \cdots$,

both series being continued as long as the arguments remain non-negative. In the case $m \equiv 3(\bmod 4)$ we have

$$
-\sum_{(a)} N\left(m-x^{2}\right)=\sum_{(b)}(-1)^{x} N\left(m-x^{2}\right),
$$

(a) $x= \pm 1, \pm 3, \pm 5, \cdots$;

(b) $x=0, \pm 1, \pm 2, \cdots$,

because $N\left(m-4 x^{2}\right)=0$ for every $x$. The equations (12) and (13) can be condensed into one,

$$
(-1)^{(m-1) / 2} \sum_{(a)} N\left(m-x^{2}\right)=\sum_{(b)}(-1)^{x} N\left(m-x^{2}\right),
$$

(a) $x= \pm 1, \pm 3, \pm 5, \cdots$;

(b) $x=0, \pm 1, \pm 2, \cdots$,

which thus holds for every odd $m$. Now it is possible to find a priori a numerical function satisfying the same equations (11) and (14). For this purpose we take

$$
f(x)=(-1)^{(x-1) / 2}
$$

in the relation (4); the resulting equation

$$
\sum_{(a)}(-1)^{d+(\delta-1) / 2}=\sum_{(b)}(-1)^{i+(\delta-1) / 2}-\left\{\frac{1}{2}\left(1+(-1)^{s-1}\right)\right\}, n=s^{2}, s>0,
$$

(a) $n=i^{2}+2 d \delta, \delta$ odd;

(b) $n=i^{2}+d \delta, \delta$ odd,

introducing the numerical function $\rho(n)$ defined by

$$
\rho(n)=\sum(-1)^{(\delta-1) / 2}, n=d \delta, \delta \text { odd } ; \rho(0)=\frac{1}{4},
$$

leads, after a simple discussion, to the following relations:

$$
\sum_{(a)}(-1){ }^{x} \rho\left(2 m-x^{2}\right)+\sum_{(b)} \rho\left(2 m-4 x^{2}\right)=0,
$$
(a) $x=0, \pm 1, \pm 2, \cdots$,
(b) $x=0, \pm 1, \pm 2, \cdots$;

$$
(-1)^{(m-1) / 2} \sum_{(a)} \rho\left(m-x^{2}\right)=\sum_{(b)}(-1)^{x} \rho\left(m-x^{2}\right),
$$
(a) $x= \pm 1, \pm 3, \pm 5, \cdots$;
(b) $x=0, \pm 1, \pm 2, \cdots$, 
perfectly analogous to (11) and (14). Putting $w(n)=N(n)-4 \rho(n)$, we have therefore

$$
\sum_{(a)}(-1)^{x} w\left(2 m-x^{2}\right)+\sum_{(b)} w\left(2 m-4 x^{2}\right)=0
$$

(a) $x=0, \pm 1, \pm 2, \cdots$;

(b) $x=0, \pm 1, \pm 2, \cdots$;

$$
\sum_{(a)}(-1)^{x} w\left(m-x^{2}\right)=(-1)^{(m-1) / 2} \sum_{(b)} w\left(m-x^{2}\right),
$$

(a) $x=0, \pm 1, \pm 2, \cdots$;

(b) $x= \pm 1, \pm 3, \pm 5, \cdots$,

or

$$
w(2 m)+\sum_{(a)}(-1)^{x} w\left(2 m-x^{2}\right)+\sum_{(b)} w\left(2 m-4 x^{2}\right)=0,
$$

(a) $x=1,2,3, \cdots$;

(b) $x=1,2,3, \cdots$;

$$
w(m)+2 \sum_{(a)}(-1)^{x} w\left(m-x^{2}\right)-2(-1)^{(m-1) / 2} \sum_{(b)} w\left(m-x^{2}\right)=0
$$

(a) $x=1,2,3, \cdots$;

(b) $x=1,3,5, \cdots$

Moreover, it follows from the definition of $\rho(n)$ that $\rho(4 n)=\rho(n)$, so that for every $n$

$$
w(4 n)=w(n)
$$

Now $w(0)=0$ and we can establish that $w(n)=0$ for every $n$ by means of mathematical induction. For, supposing the equation $w(n)=0$ established for all $n<N$, we can prove that $w(N)=0$. First, if $N$ is divisible by 4 , we have, by $(18), w(N)=w(N / 4)=0$. If $N=2 m$, where $m$ is supposed to be odd, it follows from (16) that $w(N)=0$. Finally, for an odd $N$ the same conclusion follows from (17). The equation $w(n)=0$ being thus established, we have reached at the same time the well known result

$$
N(n)=4 \rho(n)
$$

by means of extremely simple considerations.

The number of representations by the form $x^{2}+2 y^{2}$ may be obtained in a very similar way. Denoting again by $N(n)$ the number of solutions of the equation $n=x^{2}+2 y^{2}$, we have first the obvious equation $N(2 n)=N(n)$, and the following equation can be very easily verified:

$$
N(n)=0 \quad \text { when } \quad n \equiv 5 \text { or } \equiv 7
$$

Consider now the equation

$$
m=4 x^{2}+y^{2}+2 z^{2},
$$


where $m$ is supposed to be odd; the number of its solutions is given either by the sum

$$
\text { - } \sum N\left(m-4 x^{2}\right) \quad(x=0, \pm 1, \pm 2, \cdots) \text {, }
$$

or by the sum

$$
\sum N\left(m-x^{2}\right) \quad(x= \pm 1, \pm 3, \pm 5, \cdots),
$$

so that we have

$$
\sum_{(a)} N\left(m-4 x^{2}\right)=\sum_{(b)} N\left(m-x^{2}\right),
$$

(a) $x=0, \pm 1, \pm 2, \cdots$;

(b) $x= \pm 1, \pm 3, \pm 5, \cdots$.

On the other hand, the function $\phi(n)$ defined by

$$
\begin{aligned}
\phi(n)=\sum(-1)^{(\delta-1) / 2+\left(\delta^{2}-1\right) / 8} & =\sum\left(\frac{-2}{\delta}\right), n=d \delta, \delta \text { odd }, \\
\phi(0) & =\frac{1}{2},
\end{aligned}
$$

satisfies the equation of the same form. To show this we take $f(x)=\sin (\pi x / 4)$ in the relation (4). Noticing that for any odd number $x$

$$
\sin \frac{\pi x}{4}=\frac{1}{2^{1 / 2}}(-1)^{(x-1) / 2+\left(x^{2}-1\right) / 8},
$$

it is easy to get the following two equations: for $m \equiv 1(\bmod 4)$,

$$
(-1)^{(m-1) / 4} \sum_{(a)} \phi\left(m-x^{2}\right)=\sum_{(b)}(-1)^{x} \phi\left(m-4 x^{2}\right),
$$

(a) $x= \pm 1, \pm 3, \pm 5, \cdots$

(b) $\quad x=0, \pm 1, \pm 2, \cdots$,

and for $m \equiv 3(\bmod 4)$,

$$
(-1)^{(m-3) / 4} \sum_{(a)} \phi\left(m-x^{2}\right)=\sum_{(b)}(-1)^{x} \phi\left(m-4 x^{2}\right),
$$

(a) $x= \pm 1, \pm 3, \pm 5, \cdots$;

(b) $x=0, \pm 1, \pm 2, \cdots$

But as $\phi(n)=0$ when $n \equiv 5$ or $7(\bmod 8)$, it is easy to see that

$$
\begin{aligned}
& {\left[(-1)^{(m-1) / 4+x}-1\right]_{\phi\left(m-4 x^{2}\right)}=0, \text { when } m \equiv 1(\bmod 4),} \\
& {\left[(-1)^{(m-3) / 4+x}-1\right]_{\phi\left(m-4 x^{2}\right)}=0, \text { when } m \equiv 3(\bmod 4),}
\end{aligned}
$$

whence it follows that the two preceding equations may be combined into one:

$$
\sum_{(a)} \phi\left(m-x^{2}\right)=\sum_{(b)} \phi\left(m-4 x^{2}\right),
$$
(a) $\quad x= \pm 1, \pm 3, \pm 5, \cdots$;
(b) $x=0, \pm 1, \pm 2, \cdots$, 
which is perfectly analogous to (19). Putting, therefore, $w(n)=N(n)-2 \phi(n)$, we have

$$
w(m)+2 \sum_{(a)} w\left(m-4 x^{2}\right)=2 \sum_{(b)} w\left(m-x^{2}\right),
$$

(a) $\quad x=1,2,3, \cdots$;

(b) $\quad x=1,3,5, \cdots$;

and obviously $w(2 n)=w(n)$. Now it is easy to show that, for every $n$, $w(n)=0$. For, suppose this already verified for all $n<N$. If $N$ be an even number, then $w(N)=w(N / 2)=0$. But if $N$ be an odd number, the same conclusion follows from (20). As $w(0)=0$, we have $w(n)=0$ for every $n$, that is, $N(n)=2 \phi(n)$. It remains to determine by means of analogous considerations the number of representations by the form $x^{2}+3 y^{2}$. Denoting again by $N(n)$ the number of solutions of the equation $n=x^{2}+3 y^{2}$, the following properties of $N(n)$ are almost evident. First,

$$
\begin{aligned}
& N(3 n)=N(n), \\
& N(n)=0 \text { when } n \equiv 2 \quad(\bmod 3),
\end{aligned}
$$

and furthermore, if $m$ denotes an odd number,

$$
\begin{aligned}
& N\left(2^{\alpha} m\right)=0 \text { for an odd } \alpha, \\
& N\left(2^{\alpha} m\right)=N(4 m) \text { for an even } \alpha .
\end{aligned}
$$

That the equation $N(4 m)=3 N(m)$ holds true for every odd $m$, is not so evident, but still can be easily proved. It implies that the number of solutions of the equation $4 m=x^{2}+3 y^{2}$ in odd numbers $x, y$ is twice as great as the number of all solutions of the equation $m=x^{2}+3 y^{2}$. Consider now the equation

$$
4 n=\lambda^{2}+\mu^{2}+3 \nu^{2},
$$

where $\lambda$ and $\nu$ are supposed to be odd. The number of its solutions, on the one hand, is given by

$$
\sum N\left(4 n-\lambda^{2}\right) \quad(\lambda= \pm 1, \pm 3, \pm 5, \cdots) ;
$$

on the other hand it follows from the preceding remark that the same number is given by the sum $2 \sum N\left(n-\kappa^{2}\right)$ extended over all integers $\kappa$ such that $n-\kappa^{2}$ is positive and odd. Thus we get the important equation

$$
\sum N\left(4 n-\lambda^{2}\right)=2 \sum N\left(n-\kappa^{2}\right), n-\kappa^{2} \text { odd. }
$$

We shall show now that the function $\chi(n)$ defined by

$$
\chi(n)=\Sigma\left(\frac{-3}{\delta}\right), n=d \delta, \delta \text { odd, }
$$


satisfies exactly the same equation for every $n$ non-divisible by 3 . To this end we take in the relation (8)

$$
\phi(x, y)=\sin \frac{8 \pi x}{3} \cos \frac{2 \pi y}{3},
$$

which leads to the following equation:

$$
\sum_{(a)} \sin \frac{2 \pi d}{3} \cos \frac{2 \pi \delta}{3} \cos \frac{2 \pi \lambda}{3}=2 \sum_{(b)} \sin \frac{2 \pi d}{3} \cos \frac{2 \pi \delta}{3} \cos \frac{2 \pi i}{3},
$$

(a) $\quad 4 n=\lambda^{2}+d \delta, \lambda$ odd;

(b) $\quad n=i^{2}+d \delta, d$ and $\delta$ odd.

In order to exhibit it in the simplest possible form we notice first that

$$
\begin{aligned}
& \cos \frac{2 \pi \alpha}{3}=1 \text { if } \alpha \equiv 0(\bmod 3), \\
& \cos \frac{2 \pi \alpha}{3}=-\frac{1}{2} \text { if } \alpha \neq 0(\bmod 3),
\end{aligned}
$$

and that for every odd number $x$

$$
\sin \frac{2 \pi x}{3}=\frac{3^{1 / 2}}{2}\left(\frac{-3}{x}\right)
$$

Profiting by these remarks we find for $n \equiv 1(\bmod 3)$

$$
\cos \frac{2 \pi \delta}{3} \cos \frac{2 \pi \lambda}{3}=-\frac{1}{2}, \cos \frac{2 \pi \delta}{3} \cos \frac{2 \pi i}{3}=-\frac{1}{2},
$$

so that equation (22) in this case may be written as follows:

$$
\sum \chi\left(4 n-\lambda^{2}\right)=2 \sum \chi\left(n-i^{2}\right), n-i^{2} \text { odd }
$$

For $n \equiv 2(\bmod 3)$ we have

$$
\begin{aligned}
& \cos \frac{2 \pi \delta}{3} \cos \frac{2 \pi \lambda}{3}=-\frac{1}{2} \text { or }+\frac{1}{4}, \text { if } \lambda \equiv 0 \text { or } \equiv \pm 1(\bmod 3), \\
& \cos \frac{2 \pi \delta}{3} \cos \frac{2 \pi i}{3}=-\frac{1}{2} \text { or }+\frac{1}{4}, \text { if } i \equiv 0 \text { or } \equiv \pm 1(\bmod 3),
\end{aligned}
$$

and equation (22) becomes

$$
-\sum_{(a)} \chi\left(4 n-\lambda^{2}\right)+\frac{1}{2} \sum_{(b)} \chi\left(4 n-\lambda^{2}\right)=-2 \sum_{(c)} \chi\left(n-i^{2}\right)+\sum_{(\cdot d)} \chi\left(n-i^{2}\right),
$$
(a) $\lambda=0$;
(b) $\lambda= \pm 1$
(c) $\quad i \equiv 0$;
(d) $i= \pm 1(\bmod 3)$.

But for $n \equiv 2(\bmod 3)$ it is easy to show that $\chi(n)=0$, whence it follows that the preceding equation may be also written in the form 


$$
\sum \chi\left(4 n-\lambda^{2}\right)=2 \sum \chi\left(n-i^{2}\right), n-i^{2} \text { odd } .
$$

Thus, for every $n$ non-divisible by 3 we have

$$
\sum \chi\left(4 n-\lambda^{2}\right)=2 \sum \chi\left(n-i^{2}\right), n-i^{2} \text { odd, }
$$

and, putting $w(m)=N(m)-2 \chi(m)$ for an odd $m$, we reach the analogous equation for $w(n)$,

$$
\sum w\left(4 n-\lambda^{2}\right)=2 \sum w\left(n-\kappa^{2}\right), n-\kappa^{2} \text { odd, }
$$

provided $n \equiv \pm 1(\bmod 3)$. For small odd values of $m$ we find $w(m)=0$ and to prove this in general we shall apply the process of reasoning by induction.

First we have $w(m)=0$ whenever $m \equiv 2(\bmod 3)$. Now we assume as already proved that $w(m)=0$ for all $m \equiv 3(\bmod 4)$ which are $<12 k+7$ and for all $m \equiv 1(\bmod 4)$ which are smaller than $4 k+1$. Let us take in the equation (23) $n=3 k+1$ and $n=3 k+2$ respectively; in virtue of this supposition all the terms in the second member disappear, and we get

$$
\begin{aligned}
& w(12 k+3)+w(12 k-5)+\cdots=0, \\
& w(12 k+7)+w(12 k-1)+\cdots=0 .
\end{aligned}
$$

By supposition,

so that

$$
\begin{aligned}
& w(12 k-5)=0, w(12 k-21)=0, \cdots, \\
& w(12 k-1)=0, w(12 k-17)=0, \cdots,
\end{aligned}
$$

$$
\begin{aligned}
& w(12 k+3)=w(4 k+1)=0, \\
& w(12 k+7)=0 .
\end{aligned}
$$

Taking again in equation (23) $n=3 k+4$, we get

$$
w(12 k+15)+w(12 k+7)+\cdots=0,
$$

whence

$$
w(12 k+15)=w(4 k+5)=0 .
$$

Moreover, $w(12 k+11)=0$, and consequently we have $w(m)=0$ for all $m \equiv 3(\bmod 4)$ which are $<12(k+1)+7$ and for all $m \equiv 1(\bmod 4)$ which are $<4(k+1)+1$, that is, the induction is complete and the equation $w(m)=0$ established. Thus we have $N(m)=2 \chi(m)$ for any odd $m$, while $N(4 m)=6 \chi(m)$, and in general

$$
\begin{aligned}
& N\left(2^{\alpha} m\right)=6 \chi(m), \text { when } \alpha \text { is even, }>0, \\
& N\left(2^{\alpha} m\right)=0, \text { when } \alpha \text { is odd }
\end{aligned}
$$


3. Gauss-Jacobi Theorem. Taking in equation (5) $n=8 p+3$ and

$$
f(x)=\sin \frac{\pi x}{4}
$$

we get

(a)

$$
\begin{gathered}
\sum_{(a)}(-1)^{i} \cos \frac{\pi i}{4} \sin \frac{\pi d}{4}=0, \\
n=i^{2}+d \delta, \delta \text { odd, }
\end{gathered}
$$

which is equivalent to

$$
\sum_{(a)}(-1)^{h} \phi\left(n-16 h^{2}\right)=\sum_{(b)}(-1)^{\left(i^{2}-1\right) / 8} \rho\left(\frac{n-i^{2}}{2}\right),
$$

(a) $h=0, \pm 1, \pm 2, \cdots$;

(b) $i= \pm 1, \pm 3, \pm 5, \cdots$,

$\rho(n)$ and $\phi(n)$ being taken with the same meaning as above. As $\phi\left(n-16 h^{2}\right)$ and $\rho\left[\left(n-i^{2}\right) / 2\right]$ represent respectively the numbers of solutions of the equations

$$
\begin{aligned}
n-16 h^{2} & =k^{2}+2 l^{2}, & & l \text { odd }>0, \\
n-i^{2} & =2 l^{2}+8 j^{2}, & & l \text { odd }>0,
\end{aligned}
$$

it is obvious that the preceding equation can be also written in the form

$$
\sum_{(a)}(-1)^{n}=\sum_{(b)}(-1)^{\left(i^{2}-1\right) / 8}
$$

(a) $\quad n=k^{2}+2 b^{2}+16 h^{2}$;

(b) $\quad n=i^{2}+2 l^{2}+8 j^{2}$.

Now let us consider two numerical functions $G(m)$ and $g(m)$ defined for every $m \equiv 1(\bmod 8)$ by

$$
\begin{aligned}
& G(m)=\sum(-1)^{y}, \quad m=x^{2}+16 y^{2}, \\
& g(m)=\sum(-1)^{\left(x^{2}-1\right) / 8}, \quad m=x^{2}+8 y^{2},
\end{aligned}
$$

the sums being extended over all the solutions of the corresponding equations. Introducing these functions, the relation (24) can be written as follows:

$$
\sum G\left(8 p+3-2 l^{2}\right)=\sum g\left(8 p+3-2 l^{2}\right) \quad(l=1,2,3, \cdots) ;
$$

whence it follows necessarily that

$$
G(8 p+1)=g(8 p+1),
$$

that is, for every $m \equiv 1(\bmod 8)$ we have

$$
\sum_{(a)}(-1)^{y}=\sum_{(b)}(-1)^{\left(x^{2}-1\right) / 8} \text {, }
$$
(a) $m=x^{2}+16 y^{2}$;
(b) $m=x^{2}+8 y^{2}$, 
and this constitutes one of the theorems given by Jacobi. In the particular case when $m$ is a prime number, both of the equations

$$
m=a^{2}+16 b^{2}, m=c^{2}+8 d^{2}
$$

possess only one solution in positive integers, and it follows from the preceding equality that whenever $b$ is even, $c$ is of the form $8 l \pm 1$, and whenever $b$ is odd, $c$ is of the form $8 l \pm 3$. This particular theorem was first found by Gauss and published in his first memoir on biquadratic residues.

4. Another theorem by Jacobi. Preliminary results. As the second example of the use of the same kind of reasoning we choose another more complicated theorem given by Jacobi. Certain preliminary propositions are, however, to be established first. Taking $f(x)=\sin (3 \pi x / 2)$ in $(10)$, the resulting equation may be presented as follows:

$$
\sum_{(a)}(-1)^{d+(\delta-1) / 2}=2 \sum_{(b)}(-1)^{i-1} \sin \frac{\pi \delta}{2}
$$

(a) $3 n=h^{2}+2 d \delta, h \neq \equiv 0(\bmod 3), \delta$ odd; $\quad$ (b) $n=3 i^{2}+d \delta, d \not \neq 0(\bmod 3)$, or else

$$
2 \sum(-1)^{i-1} \rho\left(n-3 i^{2}\right)=\sum(-1)^{\left(n+h^{2}\right) / 2} \rho\left(3 n-h^{2}\right),
$$

the last sum being extended over all $h$ which are not divisible by 3 and of the same parity as $n$. Suppose now that $n$ is not divisible by 3 . In this case for $h$ divisible by 3 ,

$$
\rho\left(3 n-h^{2}\right)=0,
$$

because the number $3 n-h^{2}$ contains the prime number 3 only to the first power, and therefore the summation in the right hand member of (25) may be extended over all $h$ of the same parity as $n$. Taking into account the arithmetical meaning of the function $\rho(n)$ the equation (25) leads to the relations

(a)

$$
\begin{gathered}
\sum_{(a)}(-1)^{i}=(1 / 2)(-1)^{(n-2) / 2} N\left(3 n=h^{2}+2 k^{2}+2 l^{2}\right), \\
\sum_{(a)}(-1)^{i}=(1 / 2)(-1)^{(n-1) / 2} N\left(3 n=h^{2}+2 k^{2}+2 l^{2}\right), \\
n=3 i^{2}+j^{2}+k^{2},
\end{gathered}
$$

for even and odd values of $n \equiv \pm 1(\bmod 3)$ respectively. Supposing further that $n \equiv 0(\bmod 4)$ or $n \equiv 1(\bmod 4)$ and $n \equiv 1(\bmod 3)$, the two preceding equations can be combined intc one, namely

$$
\begin{gathered}
(-1)^{n-1} \sum_{(a)}(-1)^{k}=\frac{1}{4} N\left(3 n=x^{2}+2 y^{2}+2 z^{2}\right) \\
n=9 i^{*}+j^{2}+3 k^{2}
\end{gathered}
$$


But it is easy to show that

$$
\frac{1}{4} N\left(3 n=x^{2}+2 y^{2}+2 z^{2}\right)=N\left(n=x^{2}+2 y^{2}+6 z^{2}\right),
$$

and so finally we get the first preliminary result,

$$
N\left(n=x^{2}+2 y^{2}+6 z^{2}\right)=(-1)^{n-1} \sum(-1)^{k},
$$

for every $n \equiv 1(\bmod 12)$ or $\equiv 4(\bmod 12)$.

Now we put $f(x)=\sin (2 \pi x / 3)$ in the equation (5). Denoting by $\sigma_{1}$ and $\sigma_{2}$ the two sums

$$
\begin{aligned}
& \sigma_{1}=\sum(-1)^{i} \sin \frac{2 \pi d}{3}, n=9 i^{2}+d \delta, \delta \text { odd }, \\
& \sigma_{2}=-\frac{1}{2} \sum(-1)^{i} \sin \frac{2 \pi d}{3}, n=i^{2}+d \delta, \delta \text { odd }, i \neq 0(\bmod 3),
\end{aligned}
$$

extended over all corresponding representations of $n$, we have

$$
\sigma_{1}+\sigma_{2}=\frac{3^{1 / 2}}{2}\left\{(-1)^{-1}\left(\frac{s}{3}\right) s\right\}, n=s^{2}, s>0 .
$$

By means of the known expression for the number of representations by che form $x^{2}+3 y^{2}$ it is easy to express $\sigma_{1}$ and $\sigma_{2}$ as follows. Denote by $P, Q$, $R, S, T$ the numbers of the solutions of the equations

$$
\begin{aligned}
& n=9 i^{2}+j^{2}+3 k^{2} \quad \text { with } j+k \text { odd, } \\
& n=9 i^{2}+j^{2}+3 k^{2} \quad \text { with } j+k \text { even } \\
& n=i^{2}+j^{2}+3 k^{2} \quad \text { with } j+k \text { odd and } i \neq \equiv(\bmod 3), \\
& n=i^{2}+j^{2}+3 k^{2} \quad \text { with } j+k \text { even and } i \not \equiv 0(\bmod 3), \\
& n=i^{2}+2 j^{2}+6 k^{2} ;
\end{aligned}
$$

then

$$
\begin{aligned}
& \sigma_{1}=\frac{3^{1 / 2}}{4}(-1)^{n-1}\left(P-\frac{1}{3} Q\right) \\
& \sigma_{2}=\frac{3^{1 / 2}}{8}-(-1)^{n-1}\left(-R+\frac{1}{3} S-\frac{1}{3} T\right)
\end{aligned}
$$

and consequently

$$
P-\frac{1}{2} R-\frac{1}{3} Q-\frac{1}{6} S-\frac{1}{6} T=2\left\{\left(\frac{s}{3}\right) s\right\}, n=s^{2}, s>0 .
$$


By (26),

or

$$
T=(-1)^{n-1} \sum(-1)^{k}, n=9 i^{2}+j^{2}+3 k^{2},
$$

$$
T=\sum_{(a)}(-1)^{i+n-1}+\sum_{(b)}(-1)^{i+n-1}-\sum_{(c)}(-1)^{i+n-1},
$$

(a) $\quad i \equiv n, j \equiv n$;

(b) $\quad i \equiv n-1, j \equiv n$;

(c) $i \equiv n, j \equiv n-1(\bmod 2)$

all the sums being extended over solutions of the equation $n=9 i^{2}+j^{2}+3 k^{2}$, subject to the limitations indicated below each sign of the sum. For $P, Q$, $R$ and $S$ we find easily analogous expressions

$$
P=\sum_{(a)}(-1)^{i+n-1}, \quad Q=-\sum_{(b)}(-1)^{i+n-1}-\sum_{(c)}(-1)^{i+n-1},
$$
(a) $\quad i \equiv n-1, j \equiv n$;
(b) $\quad i \equiv n, j \equiv n-1$;
(c) $i \equiv n, j \equiv n(\bmod 2)$;
$R=-\sum_{(a)}(-1)^{i+n-1}$,

$$
S=\sum_{(b)}(-1)
$$
$-\sum_{(c)}(-1)^{i+n-1}$

(b) $\quad i \equiv n-1, j \equiv n$;

(a) $\quad i \equiv n, j \equiv n-1$;

(c) $\quad i \equiv n, j \equiv n(\bmod 2)$.

Now, substituting all these expressions into (27) we get

$$
\sum_{(a)}(-1)^{i+n-1}+\sum_{(b)}(-1)^{i+n-1}=2\left\{\left(\frac{s}{3}\right) s\right\}, n=s^{2}, s>0,
$$

(a) $\quad i \equiv n-1, j \equiv n$;

(b) $i \equiv n, j \equiv n-1(\bmod 2)$,

or finally

$$
\sum_{(a)}(-1)^{i}=(-1)^{n-1} 2\left\{\left(\frac{s}{3}\right) s\right\}, n=s^{2}, s>0 \text {, }
$$

$$
n=9 i^{2}+j^{2}+3 k^{2} ; i+j \text { odd. }
$$

If, supposing $n \equiv 1(\bmod 4)$, we put in $(5) f(x)=\sin (\pi x / 2)$, the resulting equation may be easily presented as follows:

(a)

$$
\sum_{(a)}(-1)^{h}=2\left\{(-1)^{(s-1) / 2} s\right\}, n=s^{2}, s>0,
$$

$$
n=i^{2}+4 h^{2}+4 k^{2} \text {. }
$$

5. Proof of Jacobi's theorem. Denoting by $m$ any odd number we shall consider two sums

$$
\begin{aligned}
& \sigma_{1}=\sum\left(\frac{x}{3}\right) x, \\
& \sigma_{2}=\sum(-1)^{(x-1) / 2+(y-1) / 2}\left(\frac{x}{3}\right) y,
\end{aligned}
$$

extended over all solutions of the equation $4 m=x^{2}+3 y^{2}$ in odd numbers $x$ and $y$. All these solutions satisfying an additional condition $x \equiv y(\bmod 4)$ 
can be derived from the solutions of the equation $m=\xi^{2}+3 \eta^{2}$ by the substitution $x=\xi+3 \eta, y=\xi-\eta$, whence it follows that

$$
\begin{aligned}
& \sigma_{1}=2 \sum\left(\frac{\xi}{3}\right)(\xi+3 \eta)=2 \sum\left(\frac{\xi}{3}\right) \xi, \\
& \sigma_{2}=2 \sum\left(\frac{\xi}{3}\right)(\xi-\eta)=2 \sum\left(\frac{\xi}{3}\right) \xi,
\end{aligned}
$$

or

$$
\sigma_{1}=\sigma_{2} \text {. }
$$

This being established we introduce the sum

$$
S=\sum(-1)^{(x-1) / 2+2}\left(\frac{x}{3}\right)
$$

extended over all the solutions of the equation $4 n=x^{2}+3\left(y^{2}+4 z^{2}+4 t^{2}\right)$, where $x$ is supposed odd, while $n$ represents any given number. Applying (29) we readily get

$$
S=\sum(-1)^{(x-1) / 2+(y-1) / 2}\left(\frac{x}{3}\right) y, 4 n=x^{2}+3 y^{2}, x \text { odd },
$$

that is, 0 for $n$ even and $\sigma_{2}$ for $n$ odd. Since $\sigma_{2}=\sigma_{1}$, we have in all cases

$$
S=\sum\left(\frac{x}{3}\right) x, 4 n=x^{2}+3 y^{2}, x \text { odd }
$$

the right hand member being naturally 0 for an even $n$. On the other hand, we can express $S$ as follows:

$$
S=\sum \psi\left(4 n-3 y^{2}\right) \quad(y= \pm 1, \pm 3, \pm 5, \cdots),
$$

introducing the function $\psi(m)$ defined by

$$
\psi(m)=\sum(-1)^{(x-1) / 2+\nu}\left(\frac{x}{3}\right) ; m=x^{2}+12 y^{2}+12 z^{2}
$$

for all $m \equiv 1(\bmod 4)$. Comparing the two expressions for $S$ we get

$$
\sum_{(a)} \psi\left(4 n-3 y^{2}\right)=\sum_{(b)}\left(\frac{x}{3}\right) x
$$
(a) $y= \pm 1, \pm 3, \pm 5, \cdots$
(b) $4 n=x^{2}+3 y^{2}, y$ odd,

whence it follows that

$$
\psi(n)=2\left\{\left(\frac{s}{3}\right) s\right\}, n=s^{2}, s>0,
$$


for every $n \equiv 1(\bmod 4)$, or

$$
\sum_{(a)}(-1)^{(x-1) / 2+y}\left(\frac{x}{3}\right)=2\left\{\left(\frac{s}{3}\right) s\right\}, n=s^{2}, s>0,
$$

$$
n=x^{2}+12 y^{2}+12 z^{2} \text {. }
$$

The right hand member here being the same as in (28), we have

$$
\sum_{(a)}(-1)^{y}=2 \sum_{(b)}(-1)^{x+y},
$$

(a) $n=x^{2}+9 y^{2}+12 z^{2}$;

(b) $n=(6 x+1)^{2}+12 y^{2}+12 z^{2}$,

and as this equation is true for every $n \equiv 1(\bmod 12)$, it follows necessarily that

$$
(-1)^{(n-1) / 4} \sum_{(a)}(-1)^{y}=2 \sum_{(b)}(-1)^{x}
$$

(a) $\quad n=x^{2}+9 y^{2}$

(b) $\quad n=(6 x+1)^{2}+12 y^{2}$,

for every $n \equiv 1(\bmod 12)$. This is Jacobi's theorem which we had to prove.

6. Stephen Smith's theorem. An interesting theorem of the same kind, but involving an indefinite form, has been given by Stephen Smith in his Report on the theory of numbers. In order to derive this theorem by our elementary methods we need to establish a certain general relation by means of a certain device which may be useful on many occasions. Returning to the fundamental equation (2) we put $4 n$ instead of $n$ and choose for $F(x, y, z)$ either

or

$$
\begin{aligned}
& F(x, y, z)=0 \text { whenever } x \text { is odd or } y \text { even, } \\
& F(x, y, z)=f(x / 4,(y+z) / 2) \text { otherwise, }
\end{aligned}
$$

$$
\begin{aligned}
& F(x, y, z)=0 \text { whenever } x \text { is odd or } y \text { even, } \\
& F(x, y, z)=f(x / 4,(y-z) / 2) \text { otherwise, }
\end{aligned}
$$

where $f(x, y)$ is an arbitrary function odd with respect to $x$ and even with respect to $y$. This gives

$$
\sum_{(a)} f\left(\frac{d+\delta}{4}, \frac{\lambda+\frac{1}{2}(d-\delta)}{2}\right)=2 \sum_{(b)} f(d+i, \delta-d-2 i)+\left\{2 \sum_{(0)} f(s, j)\right\},
$$

(a) $4 n=\lambda^{2}+d \delta, \lambda$ odd;

(b) $n=i^{2}+d \delta, \delta$ odd;

(c) $\quad j=1,3,5, \cdots, 2 s-1, n=s^{2}, s>0$;

$$
\sum_{(a)} f\left(\frac{d+\delta}{4}, \frac{\lambda+\frac{1}{2}(\delta-d)}{2}\right)=2 \sum_{(b)} f(d+i, d)+\left\{2 \sum_{(c)} f(s, 0)\right\},
$$

(a) $\quad 4 n=\lambda^{2}+d \delta, \lambda$ odd; $\quad$ (b) $n=i^{2}+d \delta, \delta$ odd

(o) $\quad=1,3,5, \cdots, 2 s-1, n=s^{2}, s>0$, 
and as the left members are obviously equal, we get
(a)

$$
\sum_{(a)} f(d+i, \delta-d-2 i)=\sum_{(b)} f(d+i, d)+\{f f(s, 0)\}-\left\{\sum f(s, j)\right\},
$$

Now we take $n$ as an odd number and suppose that $f(x, y)=0$ whenever $y$ is odd. In this case the preceding equation becomes
(a) $n=4 h^{3}+d \delta$;

$$
\sum_{(a)} f(d+2 h, \delta-d-4 h)=\sum_{(b}(d+i, d)+\{s f(s, 0)\},
$$

We specialize this equation further by taking

$$
f(x, y)=(-1)^{(x-1) / 2} F(y / 2),
$$

where $F(y)$ is an arbitrary even function; after evident reductions we obtain

(a)

$$
\begin{gathered}
\sum_{(a)}(-1)^{(d-1) / 2+h} F\left(2 h+\frac{d-\delta}{2}\right)=\left\{(-1)^{(c-1) / 2} s F(0)\right\}, \\
n=4 h^{2}+d \delta,
\end{gathered}
$$

for every odd number $n$ and even function $F(y)$. It is remarkable, however, that this equation holds true for an absolutely arbitrary function $F(y)$ if we suppose $n \equiv 1(\bmod 4)$. Let $F(y)$ be an odd function and $F(0)=0 ; n$ being $\equiv 1(\bmod 4)$, we evidently have

$$
\begin{aligned}
\sum(-1)^{(d-1) / 2+h} F\left(2 h+\frac{d-\delta}{2}\right) & =\sum(-1)^{(\delta-1) / 2+h} F\left(2 h+\frac{\delta-d}{2}\right) \\
& =-\sum(-1)^{(d-1) / 2+h} F\left(2 h+\frac{d-\delta}{2}\right),
\end{aligned}
$$

whence

(a)

$$
\sum_{(a)}(-1)^{(d-1) / 2+h} F\left(2 h+\frac{d-\delta}{2}\right)=0,
$$

$$
n=4 h^{2}+d \delta \text {. }
$$

Now let $F(y)$ be an arbitrary function. The equation (31) being satisfied for

$$
\begin{aligned}
& F_{1}(y)=\frac{1}{2}(F(y)+F(-y)), \\
& F_{2}(y)=\frac{1}{2}(F(y)-F(-y)),
\end{aligned}
$$

it will be satisfied for their sum

$$
F_{1}(y)+F_{2}(y)=F(y) .
$$

Let $k$ denote any number satisfying the condition $4 k^{2}<n$, where we suppose 
$n \equiv 1(\bmod 4)$. Taking $n-4 k^{2}$ instead of $n$ in (31) and putting $\Phi(x-2 k, 2 k)$, where $\Phi(x, y)$ is an arbitrary function, instead of $F(x)$, we get

$$
\begin{array}{rlr}
\sum_{(a)}(-1)^{(d-1) / 2+h} \Phi\left(\frac{d-\delta}{2}+2 h-2 k, 2 k\right)=\left\{(-1)^{(\cdot-1) / 2} s \Phi(-2 k, 2 k)\right\} & \\
n-4 k^{2}=4 k^{2}+d \delta . & n-4 k^{2}=s_{1}{ }^{2} s>0 .
\end{array}
$$

Here we give to $k$ all possible values consistent with the condition $4 k^{2}<n$ and add all the resulting equations; these operations performed, we arrive at the equation

$$
\sum_{(a)}(-1)^{(d-1) / 2+h} \Phi\left(\frac{d-\delta}{2}+2 h-2 k, 2 k\right)=\sum_{(b)}(-1)^{(\&-1) / 2} s \Phi(-2 k, 2 k),
$$

(a) $n=4 h^{2}+k^{2}+d \delta$;

(b) $n=s^{2}+4 k^{2}, s>0$,

which by the substitution $y=h+k, z=h-k$ can be transformed into

$$
\sum_{(a)}(-1)(d-1) / 2+(y+z) / 2 \Phi\left(\frac{d-\delta}{2}+2 z, y-z\right)=\sum_{(b)}(-1)^{(a-1) / 2} s \Phi(-2 k, 2 k),
$$

(a) $n=2 y^{2}+2 z^{2}+d \delta, d \delta \equiv 1(\bmod 4) ;$

(b) $\quad n=s^{2}+4 k^{2}, s>0$.

Now, let us define an arbitrary function $\Phi(x, y)$ as follows:

$$
\begin{aligned}
& \Phi(x, y)=0 \text { when } x \text { is different from } c, \\
& \Phi(0, y)=f(y),
\end{aligned}
$$

where $f(y)$ is an arbitrary function of a single variable. After a simple discussion we arrive at the formula

$$
\sum_{(a)}(-1)^{(x-1) / 2+(y+z) / 2} f(y+z)=\left\{(-1)^{(s-1) / 2} s f(0)\right\}, n=s^{2}, s>0,
$$

$$
n=x^{2}+2 y^{2}-2 z^{2}, x \pm 2 z>0,
$$

where the sum in the left member extends over all representations of $n$ by the indefinite form $x^{2}+2 y^{2}-2 z^{2}$, the variables being limited by the conditions $x-2 z>0, x+2 z>0$. Putting $f(x)=(-1)^{x / 2} F(x)$, we finally get

$$
\sum_{(a)}(-1)^{(x-1) / 2} F(y+z)=\left\{(-1)^{(a-1) / 2} s F(0)\right\}, n=s^{2}, s>0,
$$

$$
n=x^{2}+2 y^{2}-2 z^{2}, x \pm 2 z>0,
$$

for $n \equiv 1(\bmod 4)$ and an absolutely arbitrary function $F(x)$. Taking here $F(x)=\cos (\pi x / 2)$, we have for $n \equiv 1(\bmod 8)$

(a)

$$
\begin{gathered}
\sum_{(a)}(-1)^{(x-1) / 2+y+z}=\left\{(-1)^{(a-1) / 2} s\right\}, n=s^{2}, s>0, \\
n=x^{2}+8 y^{2}-8 z^{2}, x \pm 4 z>0,
\end{gathered}
$$


which is equivalent to

$$
\sum_{(a)}(-1)^{y} g\left(n-8 y^{2}\right)=\left\{(-1)^{(s-1) / 2} s\right\}, n=s^{2}, s>0,
$$

(a)

$$
y=0, \pm 1, \pm 2, \cdots,
$$

where $g(n)$ represents the function defined by

$$
g(n)=\sum(-1)^{(x-1) / 2+z},
$$

the sum being extended over all the representations of $n$ by the indefinite form $x^{2}-8 z^{2}$, the variables being limited by the conditions $x+4 z>0$, $x-4 z>0$.

On the other hand, we have, by (29),

(a)

$$
\sum_{(a)}(-1)^{y+z}=2\left\{(-1)^{(a-1) / 2} s\right\}, n=s^{2}, s>0 \text {, }
$$

$$
n=x^{2}+8 y^{2}+8 z^{2}
$$

that is,

(a)

$$
\begin{gathered}
\sum_{(a)}(-1)^{y} G\left(n-8 y^{2}\right)=\left\{(-1)^{(a-1) / 2} s\right\}, n=s^{2}, s>0, \\
y=0, \pm 1, \pm 2, \cdots,
\end{gathered}
$$

where $G(n)$ is defined by

$$
G(n)=\sum(-1)^{2}, n=x^{2}+8 z^{2}, x>0 .
$$

As the left members of (33) and (34) are equal, we have

$$
\sum(-1)^{y} g\left(n-8 y^{2}\right)=\sum(-1)^{y} G\left(n-8 y^{2}\right)
$$

for every $n \equiv 1(\bmod 8)$, whence it follows necessarily that $g(n)=G(n)$ for $n \equiv 1(\bmod 8)$, or

$$
\sum_{(a)}(-1)^{(x-1) / 2+8}=\sum_{(b)}(-1)^{s},
$$
a) $n=x^{2}-88^{2}, x \pm 4 z>0$;
(b) $n=x^{2}+8 x^{2}, x>0$,

which constitutes the theorem given by Stephen Smith.

Carueton College,

Northrield, Minnesota 